\title{
PREFACE
}

\section{Preface: Aerodynamics, dynamics and control of animal (insects, birds and bats) flight}

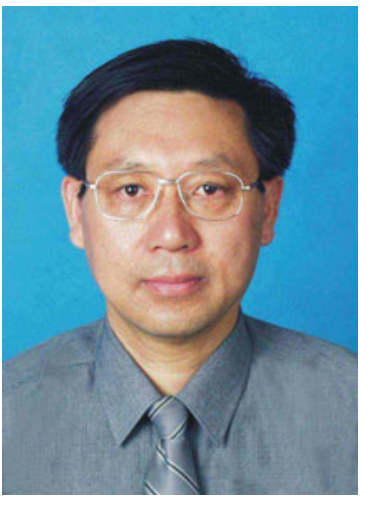

Sun Mao, Professor of the Institute of Fluid Mechanics at Beijing University of Aeronautics and Astronautics (BUAA). He graduated from BUAA in 1978, received his Ph.D. in aeronautical engineering in 1983 from Princeton University, and did post-doctorate research in University of Maryland from 1983 to 1985 . Since 1985 he has taught and conducted research at BUAA. Sun Mao's research aims to understand the intricacies of unsteady aerodynamics and animal flight dynamics through insect flight. He is studying the wing and body kinematics, unsteady aerodynamic mechanisms, energy expenditure, and flight dynamic stability and control of insect flight.

e-mail: m.sun@buaa.edu.cn
Animal flight and swimming have long been of great interest to people. Besides curiosity about how their sophisticated aero- and hydrodynamic feats are performed, researches are very interested in the mechanics of animal flight and swimming for the following two reasons. One is that biologists need to understand the effects of aero- and hydrodynamic force production and energy expenditure on the physiology, behavior, evolution and other aspects of the animals. The other is that engineers, who desire to develop tiny flying and swimming machines are eager to understand the novel aero- and hydrodynamic mechanisms of the animals and emulate their performances in the design of the small machines. Much effort has been made in this field. This theme issue of Acta Mechanica Sinica contains a number of innovative papers regarding the aerodynamics and flight dynamics of insect flight and the hydrodynamics of fish swimming from seven different groups. Three of the papers concern the aerodynamics of insect flight, dealing in turn with effects of wing aspect ratio on aerodynamics, aerodynamic interaction between forewing and hindwing of a dragonfly, and aerodynamic effects of fluid/structure interaction of a wing. Two of the papers are on flight energetics and flight dynamic stabilty of insects, respectively. The authors of the other two papers studies the hydrodynamic principles of efficient propulsion of fishes.

I expect that the papers collected in this theme issue will stimulate new thoughts in this active area. I wish to thank all the authors and reviewers for their valuable contributions. I am grateful to the staff of the editorial office of Acta Mechanica Sinica for the assistance, and I also thank the editor in chief of Acta Mechanica Sinica, Prof. Quan-Shui Zheng, for inviting me as the guest editor of this theme issue. 